Physics, Chemistry, and Dynamics of Interplanetary Dust

ASP Conference Series, Vol. 104, 1996

Bo A. S. Gustafson and Martha S. Hanner (eds.)

\title{
Isotopic Anomalies in Interplanetary Dust Particles
}

\author{
S. Messenger and R. M. Walker \\ McDonnell Center for the Space Sciences and Plysicis Department, \\ Washington University; St. Louis, MO 631.30
}

\begin{abstract}
Approximately 1/3 of interplanetary dust particles (IDPs) and some primitive meteorites have large excesses in $\mathrm{D}$ and/or ${ }^{15} \mathrm{~N}$ compared to terrestrial materials. Constraints on the nature and origin of the carriers of these isotopic anomalies are reviewed. Special attention is given to a recently discovered extremely D-rich cluster IDP.
\end{abstract}

\section{Introduction}

Large deuterium (D) enrichments in some primitive meteorites and interplanetary dust particles (IDPs) may be due to the partial preservation of presolar molecules. This interpretation arises from direct observations of extremely D-rich molecules in cold molecular clouds. These extreme D enrichments are very likely the result of 'chemical fractionation' - mass fractionation due to the difference in zero point binding erergies of $\mathrm{H}$ and $\mathrm{D}$ between reactants and products. To first order, the degree of fractionation rises exponentially with decreasing temperature. Indeed, the highest $D$ enrichments are observed in the coldest $(\sim 1() \mathrm{K})$ interstellar clouds. Chemical reactions are possible at these low temperatures if one of the reactants is ionized. Detailed models of ion-molecule chemistry have been developed that successfully reproduce the relative abundances and D enrichments of interstellar molecules (e.g., Millar et al. 1989). The study of interstellar chemistry is generally limited to spectroscopic observations of simple gas phase molecules. While some primitive meteorites have an extensive suite of D-rich organic species, the origin of these molecules appears to be primarily secondary parent body processes.

Many IDPs appear to be chemically, mineralogically and texturally primitive in comparison to meteorites. For instance, Flynn et al. (1993) have found the average volatile content of chondritic IDPs to be substantially enriched over CI levels. Many IDPs are extremely fine grained, often with highly unequilibrated mineralogy (Fraundorf 1981). Individual dust particles often have a fragile, highly porous, "flutfy" appearance, unknown among meteoritic materials. IDPs might therefore be expected to better preserve evidence of primordial or presolar chemical processes. However, by and large IDPs appear to be no more isotopically primitive than some primitive meteorites, and no identifiable circumstellar grains similar to those seen in separates from primitive meteorites have yet been seen (Anders and Zinner 1993, Walker 1994). Here we discuss a notable exception; several fragments of a large cluster IDP ( Dragonfly) have $\mathrm{D} / \mathrm{H}$ ratios enhanced by a factor of $\geq 25$ relative $t$ ) terrestrial materials. This is the highest $D$ enrichment ever observed in natural solar system materials, and reaches the level of $\mathrm{D}$ enrichments observed in interstellar molecules. 


\section{The Nature of D-Rich Phases in IDPs and Meteorites}

A thorough review on the subject of D enrichments in interplanetary dust and meteorites has been previously presented by Zinner (1988). Since that time there has been significant progress in the study of D-rich phases in meteorites, and there have been a number of new developments in the isotopic analysis of IDPs.

The first observation of D enrichments in meteorites was made by Boato (1954), who performed combustion experiments on bulk samples of carbonaceous chondrites. Subsequent work employed a variety of chemical and physical treatments on a range of primitive chondrites, in an effort to isolate the D-rich phases. D enrichments were shown to reside in organic matter by Smith and Rigby (1981) and Robert and Epstein (1982), who analyzed HF-HCL residues of carbonaceous chondrites.

Meteoritic organic matter is dominated by an acid insoluble 'kerogen'. This material is highly resistant to separation, though a number of attempts to identify subcomponents by stepwise pyrolysis or combustion have been made (e.g., Yang and Epstein 1983, 1985; Kerridge el al. 1987). In particular Kerridge et al. included H, $\mathrm{C}$, and $\mathrm{N}$ isotopic measurements for separate temperature steps and identified several isotopically distinct components. Isotopic analysis can offer strong constraints on potential formation mechanisms, though without chemically analyzing these components it is difficult to draw final conclusions.

Although a relatively small fraction $(\$ 10 \%)$ of meteoritic organic matter is solvent extractable, well over 400 individual compounds have been identified (see Cronin et al. 1988 for review). A number of these compounds have recently been subject to isotopic analysis, including (hydroxy, dicarboxylic and hydroxydicarboxylic) acids (Cronin et al. 1993), amino acids (Pizzarello et al. 1991), and (aliphatic, aromatic and polar) hydrocarbons and monocarboxylic acids (Krishnamurthy et al. 1992). To date, every separated compound subject to analysis has exhibited D enrichments to varying degrees, up to $\delta \mathrm{D}=2,500 \%$ for amino acids.

The first $\mathrm{H}$ isotopic measurements of IDPs were performed by Zinner et al. (1983) and McKeegan et al. (1985), using a modified Cameca IMS-3f ion microprobe. McKeegan et al. (1987) combined isotopic measurements with bulk mineralogy as determined by Fourier Transform Infrared (FTIR) spectroscopy in 20 IDPs. All but one of these particles fell into either a pyroxene dominated, or layer-lattice silicate spectral class (Sandford and Walker 1985). Roughly 1/3 of the particles analyzed had bulk D excesses $\geq 100 \%$, ranging up to $2,700 \%$. D enrichments occurred in roughly equal numbers in the anhydrous and hydrated FTIR classes.

Although equally large bulk D enrichments occur in some primitive meteorites, IDPs show $\mathrm{H}$ isotopic heterogeneity on a $\mu h \mathrm{~m}$ size scale not yet seen in meteorites. The most extreme example of this is the IDP Butterfly (McKeegan et al. 1987). Individually measured subfragments yielded $\delta \mathrm{D}$ values between -320 and $+2,700 \%$. Ion imaging of one D-rich fragment showed that the spatial distribution of the D was strongly concentrated relative to the $\mathrm{H}$. The $\delta \mathrm{D}$ in this $\leqslant 1 \mu \mathrm{m}$ D-rich "hot spot" was estimated to be at least $9,000 \%$.

Recent isotopic measurements of fragments from a large 'cluster' IDP ('Dragonfly'; Messenger et al. 1995) dramatically surpass the earlier results. Bulk D excesses between $6(0)$ and $24,800 \%$ were observed among the fragments. Ion imaging of one fragment found the $\mathrm{D}$ concentrated relative to the $\mathrm{H}$, though not as extreme as observed in Butterfly. The D excess in this fragment is estimated to reach $50,0()(0 \%)$. 
Characterizing the D-rich phases in IDPs is difficult because of their small size ( $\sim 1$ nanogram). However when it has been possible to make D/H measurements on different subfragments of a given IDP, D enrichments has have positively correlated with the C/O ratio (McKeegan et al. 1987). The D-rich phases are therefore inferred to be carbonaceous, although the molecular nature of this material remains unknown. The first direct measurements of organic molecules in IDPs, specifically polycyclic aromatic hydrocarbons (PAHs), have been recently reported by Clemett et al. (1993). While it is not yet possible to directly determine the $\mathrm{H}$ isotopic compositions of these molecules, it is interesting to note that of the 8 IDPs yielding strong PAH signals, so far all have D enrichments, although the converse is not true.

\section{Possible Correlations With Other Isotopic Anomalies}

The large and variable D excesses found in meteoritic organic matter and IDPs are not accompanied by substantial $\mathrm{C}$ isotopic anomalies. The $\mathrm{C}$ isotopic composition of acid residues and soluble organics lies close to $\delta^{13} \mathrm{C}=-15 \%$. Although the observed range of $\delta^{13} \mathrm{C}$ is only $\sim 40 \%$, Kerridge et al. (1987) have reported several isotopically distinct components among the meteoritic kerogen. There is a similar range in $\delta^{13} \mathrm{C}$ among IDPs, though no correlation with $\delta$ D has been observed (McKeegan et al. 1985).

In contrast to their essentially normal $\mathrm{C}$ isotopic compositions, substantial ${ }^{15} \mathrm{~N}$ excesses have been observed in both meteoritic organic matter and IDPs, though the size of these effects is much smaller than the corresponding D enrichments. The largest reported $\delta^{15} \mathrm{~N}$ excess in meteoritic organics (in Inman residues) is $256 \%$ (Alexander et al. 1989). There does not appear to be any simple correlation between $\delta \mathrm{D}$ and $\delta^{15} \mathrm{~N}$ in either organic extracts or kerogen, with the exception of CR chondrites (Ash et al. 1993). Kerridge (1985) suggested a possible positive correlation in whole rock measurements of 25 meterites, though there appears to be an anticorrelation among $\mathrm{CO}$ and $\mathrm{CV}$ chondrites.

Bulk ${ }^{15} \mathrm{~N}$ excesses are observed in almost half of chondritic IDPs analyzed (Stadermann et al. 1989), and can be much higher than those observed in meteoritic organics. Although particles with large ${ }^{15} \mathrm{~N}$ excesses are usually D-rich (indeed, Dragonfly also has the largest $\delta^{15} \mathrm{~N}$ yet measured, $+48(0 \%)$, the converse is not necessarily true, and there is no clear quantitative correlation between the effects.

\section{The Origin of $\mathrm{D}$ and ${ }^{15} \mathrm{~N}$ Excesses in IDPs and Meteorites}

Geiss and Reeves (1981) considered the origin of D enrichments in primitive meteorites, concluding that they were the result of ion-molecule chemistry at low temperatures, under conditions characteristic of interstellar molecular clouds. A solar nebula origin was ruled out due to expected low ionization levels in regions cold enough for such large fractionation effects. It is interesting to reconsider the issue in light of the extreme D enrichments observed in the IDP Dragonfly.

We can estimate an upper limit on the formation temperature of the most D-rich material in Dragonfly by assuming the highest known exothermic exchange reaction: $\mathrm{C}_{2} \mathrm{H}_{2}{ }^{+}+\mathrm{HD} \rightarrow \mathrm{C}_{2} \mathrm{HD}^{+}+\mathrm{H}_{2}$, with an exothermicity of $550 \mathrm{~K}$. Assuming equilibrium fractionation, we find an upper limit formation temperature of $70 \mathrm{~K}$. N isotopic fractionation is also expected to occur at low temperatures, though to a much smaller degree 
than the corresponding D enrichments (Adams and Smith, 1981). The ${ }^{15} \mathrm{~N}$ enrichmer observed in Dragonfly would require a formation temperature of $<2() \mathrm{K}$. There is $\mathrm{n}$ yet strong observational support for $\mathrm{N}$ isotopic fractionation in cold molecular clouc though the number and precision of such measurements is small.

The large $\mathrm{D}$ and ${ }^{15} \mathrm{~N}$ enrichments are thus consistent with the hypothesis that ID: contain preserved material synthesized in cold molecular clouds.

\section{References}

Adams, N.G., \& Smith, D. 1981, ApJ, 247, L123

Alexander, C.M.O'D. et al. 1989, Lunar Planet. Sci. XX, 7

Anders, E., \& Zinner, E. 1993, Meteoritics, 28, 49()

Ash, R.D. et al. 1993, Meteoritics, 28, 318

Boato, G. 1954, Geochim. Cosmochim. Acta, 6, 209

Clemett, S.J. et al. 1993, Science, 262, 721

Cronin, J.R. et al. 1988, in Meteorites and the Early Solar System, ed. J.F. Kerridge M.S. Matthews (Tucson: Univ. of Arizona Press), 819

Flynn, G.J. et al. 1993, Lunar Planet. Sci. XXIV, 495

Fraundorf, P, 1981, Geochim. Cosmochim. Acta, 45, 915

Geiss, J., \& Reeves, H. 1981, A\&A, 93, 189

Kerridge, J.F. 1985, Geochim. Cosmochim. Acta, 49, 1707

Kerridge, J.F et al. 1987, Geochim. Cosmochim. Acta, 51, 2527

Krishnamurthy R. V. et al. 1992. Geochim. Cosmochim. Acta, 56, 4()45

McKeegan, K.D. et al. 1985, Geochim. Cosmochim. Acta, 49, 1971

McKeegan, K.D. et al. 1987, Lunar Planet. Sci. XVIII, 627

Messenger, S. et al. 1995, Meteoritics, 30, 546

Millar, T.J. et al. 1989, ApJ, 34(), 9()6

Pizzarello, S. ct al. 1991, Geochim. Cosmochim. Acta, 55, 905

Robert, F., \& Epstein, S. 1982, Geochim. Cosmochim. Acta, 46, 81

Sandford, S., \& Walker. R.M. 1985, ApJ, 291, 838

Stadermann, F.J et al. 1989, Meteoritics, 24, 327

Smith, J.W., \& Rigby, D. 1981, Earth Planet. Sci. Lett., 54, 64

Walker, R.M. 1994, in proc. Workshop in the Analy sis of Interplanetary Dust Particle 2() 3

Yang, J.. \& Epstcin, S. 1983, Geochim. Cosmochim. Acta, 47, 2199

Yang, J., \& Epstein, S. 1985, Gcophys. Res. Lett. 12, 73

Zinner, E. 1988, in Meteorites and the Early Solar System, 956

Zinner, E. et al. 1983, Nature, 305. 119 